\title{
Cyanobacterial $\mathrm{CO}_{2}$-concentrating mechanism components: function and prospects for plant metabolic engineering
}

BENEDICT M. LONG ${ }^{1}$, BENJAMIN D. RAE ${ }^{1}$, VIVIEN ROLLAND ${ }^{1,2}$, BRITTA FÖRSTER ${ }^{1}$, AND G. DEAN PRICE ${ }^{1}$

\author{
${ }^{1}$ ARC Centre of Excellence for Translational Photosynthesis, Division of Plant Sciences, Research \\ School of Biology, Australian National University, Canberra, ACT, 2601, AUSTRALIA \\ ${ }^{2}$ Present address: CSIRO Agriculture, GPO Box 1600, Canberra ACT 2601, AUSTRALIA
}

Author for correspondence: B. M. Long, Fax:+61-261250313; Email: ben.long@anu.edu.au

Running head: Cyanobacterial CCMs for photosynthetic improvement

$\begin{array}{llll}\text { Author details: } & \text { ORCID } & \text { ResearcherID } & \text { Email address } \\ \text { B. M. Long } & 0000-0002-4616-2967 & \text { B-3799-2008 } & \text { ben.long@anu.edu.au } \\ \text { B. D. Rae } & 0000-0003-2199-1205 & \text { C-3045-2011 } & \text { benjamin.rae@anu.edu.au } \\ \text { V. Rolland } & 0000-0002-9232-1287 & \text { 0-9126-2015 } & \text { vivien.rolland@csiro.au } \\ \text { B. Förster } & 0000-0002-5231-0954 & \text { 0-7908-2015 } & \text { britta.forster@anu.edu.au } \\ \text { G. D. Price } & 0000-0001-5906-4912 & \text { C-9505-2009 } & \text { dean.price@anu.edu.au }\end{array}$




\begin{abstract}
Global population growth is projected to outpace plant-breeding improvements in major crop yields within decades. To ensure future food security, multiple creative efforts seek to overcome limitations to crop yield. Perhaps the greatest limitation to increased crop yield is photosynthetic inefficiency, particularly in $C_{3}$ crop plants. Recently, great strides have been made toward crop improvement by researchers seeking to introduce the cyanobacterial $\mathrm{CO}_{2}$ concentrating mechanism (CCM) into plant chloroplasts. This strategy recognises the $\mathrm{C}_{3}$ chloroplast as lacking a CCM, and being a primordial cyanobacterium at its essence. Hence the collection of solute transporters, enzymes, and physical structures that make cyanobacterial $\mathrm{CO}_{2}$-fixation so efficient are viewed as a natural source of genetic material for $\mathrm{C}_{3}$ chloroplast improvement. Also we highlight recent outstanding research aimed toward the goal of introducing a cyanobacterial CCM into $\mathrm{C}_{3}$ chloroplasts and consider future research directions.
\end{abstract}


2 A prime limitation of plant photosynthetic carbon acquisition is the enzyme D-ribulose-1, 5bisphosphate carboxylase/oxygenase (RuBisCO), whose slow kinetics and poor discrimination between $\mathrm{CO}_{2}$ and $\mathrm{O}_{2}$ as substrates make it an ideal target for genetic improvement in crop plants [1]. In addition, $\mathrm{C}_{3}$ chloroplastic $\mathrm{CO}_{2}$ concentrations typically fall below the $K_{\mathrm{M}} \mathrm{CO}_{2}$ of $\mathrm{RuBisCO}$, often leaving photosynthetic carbon acquisition limited by this critical carbon-fixation step. To overcome RuBisCO's limitations, a number of terrestrial plant species have evolved $\mathrm{CO}_{2}$ concentrating mechanisms (CCMs), including $\mathrm{C}_{4}$ photosynthesis and crassulacean-acid metabolism, which elevate $\mathrm{CO}_{2}$ concentrations near RuBisCO to enhance $\mathrm{CO}_{2}$-fixation [2]. In aquatic environments, photosynthetic organisms (predominantly microalgae and cyanobacteria) have evolved highly effective CCMs that rely on a range of active and facilitated uptake systems for inorganic carbon $\left(\mathrm{C}_{\mathrm{i}} ; \mathrm{HCO}_{3}{ }^{-}, \mathrm{CO}_{2}\right)$ to enhance photosynthetic $\mathrm{CO}_{2}$-fixation [3]. Most of the world's major staple crops perform $C_{3}$ photosynthesis, suggesting improvement of net RuBisCO carboxylation rates is a way forward to increased yield potential, urgently needed for future food security [4]. It is expected, based on photosynthetic modelling, that a $\mathrm{C}_{3}$ chloroplast possessing a cyanobacterial CCM will provide significant improvements in photosynthetic performance and yields $[5,6]$. Toward this goal, herein we discuss the current progress, engineering requirements and limitations in current knowledge of cyanobacterial CCMs.

Cyanobacterial CCMs include energised bicarbonate transporters, $\mathrm{CO}_{2}$-uptake complexes [7], and ancillary and regulatory proteins [8] which elevate the cytoplasmic $\mathrm{C}_{\mathrm{i}}$ pool, primarily as less membrane-permeable bicarbonate ion (Figure 1). Cytoplasmic bicarbonate is transferred across a selectively-permeable protein shell into the carboxysome, a RuBisCO micro-compartment $[9,10]$, where it is dehydrated to $\mathrm{CO}_{2}$ and incorporated into 3-phosphoglycerate (Figure 1) $[9,10]$. This arrangement favours cyanobacterial RuBisCOs, which have high enzyme fluxes (high $V_{\mathrm{CO}_{2}}$ ) compared with higher plant RuBisCOs, but at the cost of substrate affinity (high $K_{\mathrm{M}}{ }^{\mathrm{CO}_{2}}$ ) [11]. Elevated $\mathrm{CO}_{2}$ within the carboxysome enables high kinetic turnover, minimizing photorespiration.

Prior work from our laboratory has outlined a hypothetical $\mathrm{C}_{3}$ chloroplastic CCM incorporating cyanobacterial components [6,12]. A first-order analysis reveals an engineering trajectory (further outlined in Figure 2):

1. Introduction of active bicarbonate transporters and potential ancillary systems

2. Introduction of carboxysomes and carboxysomal RuBisCO 
Mathematical modelling shows that substantial increases in substrate-saturated $\mathrm{CO}_{2}$ assimilation rate $\left(A_{\text {sat }}\right)$ and yield can be achieved $\left(A_{\text {sat }}\right.$ increase up to $60 \%$; $36-60 \%$ yield increase) by incorporation of a cyanobacterial CCM in $\mathrm{C}_{3}$ chloroplasts [5]. At minimum, photosynthetic improvements can be made by incorporation of functional cyanobacterial bicarbonate transporters in the chloroplast inner membrane. However, maximum improvements will only be achieved by incorporation of further components of a complete CCM (Figure 1; Figure 2; Table 1) and genetic deletion of stromal carbonic anhydrase (CA) [5,6]. The importance of ancillary systems involved in aiding $C_{i}$ uptake by maintenance of electrochemical gradients (Figure 1) is currently either unknown or unclear [6] and will not be discussed further. The bicarbonate transporters, BicA and SbtA (Figure 1), have been suggested as primary candidates for chloroplast engineering since they likely function as homomeric complexes, thus eliminating the need to express and assemble different protein subunits in correct stoichiometry. In contrast, engineering of multi-component $C_{i}$ uptake systems (Figure 1) such as BCT1, NDH-I $\mathrm{I}_{3}$ and $\mathrm{NDH}-\mathrm{I}_{4}$ (NADPH dependent, $\mathrm{CO}_{2}$ uptake complexes) will only marginally increase photosynthesis [5], but add the complication of coordinated expression of both membrane-associated and cytosolic proteins. While the construction of a fully-functional carboxysome in the chloroplast stroma is equally challenging, early steps have recently been made $[13,14]$. We discuss here some of the recent advances, remaining questions and constraints toward carboxysome formation in $\mathrm{C}_{3}$ chloroplasts.

\section{Challenges of expressing functional bicarbonate transporters in chloroplasts}

A milestone toward the generation of a chloroplastic CCM was the successful expression of the cyanobacterial bicarbonate transporter BicA in chloroplasts of Nicotiana tabacum via plastome transformation [15]. However, predominant localization of BicA to the thylakoids, and lack of function, highlights the challenges in correct targeting and activation of foreign transporters in chloroplasts. Indeed, BicA expressed in E. coli [16] or in Xenopus oocytes (Förster, B., and Price, G. D., unpublished) is also inactive. It is therefore paramount to understand the activation mechanism in order to use BicA effectively in the chloroplast envelope. While plastomeencoding alleviates the need for organellar targeting peptides, the fact that most $\mathrm{C}_{3}$ crop plants are recalcitrant to plastid transformation makes expression from the nucleus a promising alternative. 
To be functional in higher plants, nuclear-encoded cyanobacterial bicarbonate transporters BicA and SbtA need to be: (1) efficiently targeted to chloroplasts, (2) inserted in the correct membrane, (3) in an orientation allowing transport of bicarbonate into chloroplasts, and (4) active and regulated.

\section{Chloroplast targeting and orientation in the envelope}

Chloroplast protein import is well understood, and generally relies on a cleavable chloroplast transit peptide (cTP) [17]. Efficient import of foreign proteins is more difficult and until recently data only existed for soluble proteins, in which case both a cTP and part of the mature protein are required (a combination called a transit peptide, TP) [18,19]. No "universal" TP able to deliver any cargo to the chloroplast is known, and instead TPs are likely to be cargo-specific. In fact, a recent study showed that TPs used for stromal cargos were too short to target large transmembrane proteins such as BicA and SbtA, to the inner envelope membrane. However, longer TPs were sufficient for chloroplast targeting, without the need for extra transmembrane domains [20]. In a separate study, BicA and SbtA were targeted to chloroplasts by fusion to another transmembrane protein which could be cleaved off post-import [21].

Chloroplasts contain three membranous compartments: the outer- and inner-envelope membranes (OEM and IEM), and the thylakoids. The OEM is a porous membrane and, to present knowledge, only one of its proteins contains an identifiable cTP [22]. The IEM is the selective membrane in which most of the transporters regulating solute fluxes are located $[23,24]$, and is the proposed membrane in which BicA and SbtA need to be localized in order to effect bicarbonate transport [12]. Overexpression of proteins in the IEM or OEM result in differentlyshaped membrane out-foldings (stromules), suggesting it is possible to identify the membrane containing the protein of interest [25].

The thylakoids are fused at focal points with the IEM, such that the IEM-thylakoids form a continuous membrane [26], and protein movement between the two membranes is not well understood. However, the membrane domain of proteins with a single trans-membrane-domain (TMD) contains sufficient information to target it to the IEM or the thylakoid [27]. The case of foreign proteins with multiple TMDs such as BicA and SbtA, which have evolved to be localised in the plasma membrane in cyanobacteria [7] is likely to be more complex [20].

BicA and SbtA likely follow the positive-inside rule governing their orientation in the plasma membrane [28,29], but it is unknown how foreign proteins insert into the plastid IEM. Therefore it is critical to assess protein orientation in vivo. As described recently, a self- 
assembling split GFP-fluorophore may be used to unravel the orientation of transporters in the IEM [30-33].

\section{Regulation of BicA and SbtA transport activity}

Rapid regulation in response to $C_{i}$ availability and light is a common feature of all $C_{i}$ transporters [7]. However, the underlying post-translational regulatory mechanisms in cyanobacteria are poorly understood. Insights from heterologous expression in E. coli suggest that SbtA may be directly regulated by a companion protein SbtB [16]. A mechanism for SbtA SbtB interaction has been proposed based on similarities of an unpublished SbtB crystal structure from Anabaena (PDB: 3DFE), with cyanobacterial PII proteins that regulate nitrogen metabolism [16, and references therein]. By analogy to the regulation of the E. coli ammonia channel AmtB by its PII protein counterpart GlnK [34], it is conceivable that small-molecule cofactors may mediate a reversible binding of SbtB to SbtA. Thus, we view SbtA as being manageable once expressed in the chloroplast envelope.

Although we presently have no experimental evidence for SbtA or BicA function in higher plants, it is promising that several algal bicarbonate transporters localise to equivalent subcellular compartments both in from Chlamydomonas and higher plants [35]. Two of those transporters (LCIA and HLA3) facilitate bicarbonate uptake into Xenopus oocytes, albeit exerting no measurable phenotype in planta, re-iterating the necessity to understand regulation of transporters and the need for highly sensitive assays for bicarbonate transporter activity in plant cells.

\section{Carboxysomes: Expression and Engineering in $\mathrm{C}_{3}$ Chloroplasts - current advances}

Carboxysomes are protein mega-complexes containing the cellular RuBisCO and CA enzymes in model species $[9,10]$ (and elsewhere in this issue, Kerfeld, C.). Formation of these bodies in cyanobacteria and Proteobacteria relies on the stoichiometric expression of as few as eight, or as many as 13 genes (Figure 3). Two architecturally distinct types of carboxysomes are evident; $\alpha$-carboxysomes possess bacterial RuBisCO form-1A, whereas $\beta$-carboxysomes encapsulate higher-plant RuBisCO form-1B [36]. Both types utilise many thousands of homologous proteins in tessellated arrays forming an icosahedral outer shell structure [37]. The outer shell is selectively permeable, allowing transit of RuBisCO substrates and the bicarbonate ion, but limited $\mathrm{CO}_{2}$ and $\mathrm{O}_{2}$ diffusion (Figure 1; Table 1) [38]. 
Additional to the challenge of successful carboxysome construction in the chloroplast stroma is 130 the expression of a fully functional, foreign, carboxysomal RuBisCO [11]. Attempts at ectopic expression of RuBisCO proteins have been hampered by the complex chaperone requirements of this enzyme [11]. Depending on the species of origin, numerous RuBisCO chaperones are utilised (Table 1) [39-41]. The extent to which these chaperone requirements can be satisfied by the $\mathrm{C}_{3}$ chloroplast remains a topic of extensive research, as is the chaperone requirement of bona fide $\mathrm{C}_{3}$ RuBisCO enzymes [42]. While numerous barriers prevent the effective expression of even closely related RuBisCO enzymes in $\mathrm{C}_{3}$ chloroplasts, recent advances suggest cognate chaperones are required [43]. Further studies have also elaborated the roles of $\alpha$-carboxysomespecific RuBisCO chaperones and activases acRAF, CbbQ-CbbO, and CbbX (Table 1) $[40,41,44,45]$. On the other hand, $\beta$-carboxysomal RuBisCO requires the Rca activase (Table 1 ) [46], but not RbcX when co-expressed with RuBisCO in N. tabacum [14].

\section{Structural and ancillary proteins}

142 Gross differences in structure exist among carboxysomes (Table 1). Both types utilise a CA 143 enzyme, however $\beta$-carboxysomes may use either of two genes for this purpose [47]. The main body of RuBisCO enzymes is arranged differently in $\alpha$ - and $\beta$-carboxysomes [recently reviewed in depth, 9]. RuBisCO is attached to the shell via CsoS2 proteins in $\alpha$-carboxysomes $[48,49]$, whereas in $\beta$-carboxysomes RuBisCO enzymes are scaffolded to one another, and to $\mathrm{CA}$ enzymes, by CcmM proteins [50,51]; the shell is attached to this body via another structural protein CcmN [52]. Lin, et al. [13] successfully co-expressed CcmM and RuBisCO from $S$. elongatus PCC 7942 in chloroplasts of N. tabacum, resulting in apparent macromolecular complexes, in some ways reminiscent of structures upon which $\beta$-carboxysomal models are based [53-55]. However, similar to transgenic plants expressing form II RuBisCO in chloroplasts [56], those generated by Lin, et al. [13] could not grow without $\mathrm{CO}_{2}$ supplementation, highlighting the important fact that we do not expect cyanobacterial RuBisCO alone, or in the carboxysome, to operate effectively in the absence of bicarbonate transport or stromal CA removal $[5,6]$.

\section{Complex carboxysome genetics}

157 Carboxysomes with the smallest genetic footprint are the most appealing for $\mathrm{C}_{3}$ chloroplast 158 transformation (Figure 3). With lower genetic load, and minimal potential for recombination 159 between homologous shell genes, we expect that simple carboxysomes might be better able to 160 self-assemble in chloroplasts. While $\alpha$ and $\beta$-carboxysomes appear to assemble from their 161 components with remarkable ease, complex expression of the major organising proteins of both 162 types is troubling. Two forms of $\mathrm{CcmM}$ are present in $\beta$-carboxysomes, with a short form arising 
from an internal ribosome-entry site within ccmM [50]. Two isoforms of the $\alpha$-carboxysomal protein CsoS2 arise from the gene via translational frame-shifting in many organisms [49]. Both forms of CcmM are essential to the functioning of the $\beta$-carboxysome [54], hence it must be ascertained whether these can be accurately produced in $\mathrm{C}_{3}$ chloroplasts. On the other hand, it appears that only the long-form of CsoS2 is truly essential in $\alpha$-carboxysomes [49].

To achieve similar goals, Gonzalez-Esquer, et al. [57] generated carboxysome-like bodies from a single carboxysomal fusion-protein, $\mathrm{CcmC}$ (containing shell, $\mathrm{CA}$, and RuBisCO-binding domains). Further work is required to align the physiology with the observed structure, but we recognise this innovative work to have significantly advanced the field.

\section{Ideal carboxysomes from genomic data}

Candidate operons encoding the minimal gene requirements for both $\alpha$ - and $\beta$-carboxysomes formation exist (Figure 3): Gloeobacter violaceus PCC 7421 has the smallest known $\beta$ carboxysome gene content, encoding just five shell proteins, and utilising CcmM as its CA enzyme [47]. Candidate $\alpha$-carboxysome operons are evident in the high-light adapted Prochlorococcus marinus clade [58], possessing the simplest known $\alpha$-carboxysome operons, and also likely to use a single form of CsoS2 $[49,58]$. Typically, we recognise $\alpha$-carboxysomes as most desirable in terms of gene content, self-assembly in transgenic hosts, and simplicity of gene expression.

Because $C_{3}$ carboxysome models are sensitive to RuBisCo kinetics [5], we must take into account the varied kinetics observed in extant RuBisCO enzymes. Essentially, a carboxysomal RuBisCO should operate near its maximum rate of catalysis. Therefore, $\beta$-carboxysomal RuBisCO enzymes, being catalytically superior to $\alpha$-carboxysomal homologues [59-65] might be preferred. Interestingly, the Cyanobium genus possess a high affinity CCM and fast $\alpha$ carboxysomal RuBisCO [62,66], overcoming this shortfall, hence we view Cyanobium carboxysomes as bearing many desirable traits for chloroplast engineering. Nonetheless, a dearth of kinetic data for RuBisCOs from $\alpha$-carboxysomes suggests that an ideal $\alpha$-carboxysomal RuBisCO candidate for a chloroplastic CCM is yet to be identified.

\section{Conclusions}

Recent advances toward a chloroplastic CCM [13-15,35] are vital first steps, but highlight shortcomings in achieving this goal. These developments emphasise the need to examine targeting and regulation (in the case of $\mathrm{C}_{\mathrm{i}}$ transporters), and issues of protein folding and 
195 activity (in the case of carboxysomes). Indeed, our efforts focus on engineering $\mathrm{C}_{3}$ chloroplasts

196 with simple cyanobacterial systems, such as the single-subunit $\mathrm{C}_{\mathrm{i}}$ transporters BicA and SbtA in

197 the hope that regulation is achievable. This extends to genetically simple carboxysomes, which

198 are apparent from both types (Figure 3). The difficult nature of $\beta$-carboxysome purification [67]

199 makes them problematic in confirming their functional presence in transgenic plants. Whereas,

$200 \alpha$-carboxysomes are readily expressed in, and purified from, tractable bacterial and

201 cyanobacterial systems [48,58,68]. Despite the catalytic superiority of $\beta$-carboxysomal

202 RuBisCOs, we identify a lack of data for $\alpha$-carboxysomal RuBisCOs and work in this field is

203 needed. In what is a rapidly developing field of research, our expectations for progress toward a

204 chloroplastic CCM utilising cyanobacterial components are optimistic.

205

\section{Acknowledgements}

207 We thank Murray Badger for comments on the manuscript. Our work is funded via the 208 University of Illinois as part of the Bill and Melinda Gates Foundation-funded Realizing 209 Increased Photosynthetic Efficiency (RIPE) consortium, and the Australian Research Council's

210 Centre of Excellence for Translational Photosynthesis. 
212

213

214

215

216

217

218

219

220

221

222

223

224

225

226

227

228

229

230

231

232

233

234

235

236

237

238

239

240

241

242

243

244

245

246

247

248

249

250

251

252

253

254

255

256

257

258

259

260

261

1. Furbank RT, Quick WP, Sirault XRR: Improving photosynthesis and yield potential in cereal crops by targeted genetic manipulation: Prospects, progress and challenges. Field Crops Research 2015, 182:19-29. 10.1016/j.fcr.2015.04.009

2. Sage RF: Photosynthetic efficiency and carbon concentration in terrestrial plants: the $\mathbf{C}_{\mathbf{4}}$ and CAM solutions. J Exp Bot 2014, 65:3323-3325. 10.1093/jxb/eru262

3. Raven JA, Giordano M, Beardall J, Maberly SC: Algal evolution in relation to atmospheric $\mathrm{CO}_{2}$ : carboxylases, carbon-concentrating mechanisms and carbon oxidation cycles. Philos Trans R Soc Lond B Biol Sci 2012, 367:493-507. 10.1098/rstb.2011.0212

4. Parry MA, Andralojc PJ, Scales JC, Salvucci ME, Carmo-Silva AE, Alonso H, Whitney SM: Rubisco activity and regulation as targets for crop improvement. J Exp Bot 2013, 64:717-730. 10.1093/jxb/ers336

5. McGrath JM, Long SP: Can the cyanobacterial carbon-concentrating mechanism increase photosynthesis in crop species? A theoretical analysis. Plant Physiol 2014, 164:2247-2261. 10.1104/pp.113.232611

- This important modelling paper analyses the predicted effects of the addition of cyanobacterial CCM components to $\mathrm{C}_{3}$ plant chloroplasts on both photosynthetic carbon acquisition and yield. The modelling shows that significant improvements in both photosynthetic parameters are to be expected.

6. Price GD, Pengelly JJ, Forster B, Du J, Whitney SM, von Caemmerer S, Badger MR, Howitt SM, Evans JR: The cyanobacterial CCM as a source of genes for improving photosynthetic $\mathrm{CO}_{2}$ fixation in crop species. J Exp Bot 2013, 64:753-768. $10.1093 / \mathrm{jxb} / \mathrm{ers} 257$

7. Price GD: Inorganic carbon transporters of the cyanobacterial $\mathrm{CO}_{2}$ concentrating mechanism. Photosynth Res 2011, 109:47-57. 10.1007/s11120-010-9608-y

8. Price GD, Badger MR, Woodger FJ, Long BM: Advances in understanding the cyanobacterial $\mathrm{CO}_{2}$-concentrating-mechanism (CCM): functional components, $\mathrm{Ci}$ transporters, diversity, genetic regulation and prospects for engineering into plants. J Exp Bot 2008, 59:1441-1461. 10.1093/jxb/erm112

9. Rae BD, Long BM, Badger MR, Price GD: Functions, compositions, and evolution of the two types of carboxysomes: polyhedral microcompartments that facilitate $\mathrm{CO}_{2}$ fixation in cyanobacteria and some proteobacteria. Microbiol Mol Biol Rev 2013, 77:357-379. 10.1128/MMBR.00061-12

10. Rae BD, Long BM, Whitehead LF, Forster B, Badger MR, Price GD: Cyanobacterial carboxysomes: microcompartments that facilitate $\mathrm{CO}_{2}$ fixation. $J$ Mol Microbiol Biotechnol 2013, 23:300-307. 10.1159/000351342

11. Whitney SM, Houtz RL, Alonso H: Advancing Our Understanding and Capacity to Engineer Nature's $\mathrm{CO}_{2}$-Sequestering Enzyme, Rubisco. Plant Physiol 2011, 155:2735. 10.1104/pp.110.164814

12. Price GD, Badger MR, von Caemmerer $S$ : The prospect of using cyanobacterial bicarbonate transporters to improve leaf photosynthesis in $\mathbf{C}_{3}$ crop plants. Plant Physiol 2011, 155:20-26. 10.1104/pp.110.164681

13. Lin MT, Occhialini A, Andralojc PJ, Devonshire J, Hines KM, Parry MA, Hanson MR: $\beta$ Carboxysomal proteins assemble into highly organized structures in Nicotiana chloroplasts. Plant J 2014, 79:1-12. 10.1111/tpj.12536

14. Lin MT, Occhialini A, Andralojc PJ, Parry MA, Hanson MR: A faster Rubisco with potential to increase photosynthesis in crops. Nature 2014, 513:547-550. 10.1038/nature13776

15. Pengelly JJ, Forster B, von Caemmerer S, Badger MR, Price GD, Whitney SM: Transplastomic integration of a cyanobacterial bicarbonate transporter into tobacco chloroplasts. J Exp Bot 2014, 65:3071-3080. 10.1093/jxb/eru156 
-This is the first evidence of plastomic integration of a cyanobacterial bicarbonate transporter in higher plants. However, BicA was not functional, highlighting the need to identify regulatory mechanisms underpinning its activation.

16. Du J, Forster B, Rourke L, Howitt SM, Price GD: Characterisation of cyanobacterial bicarbonate transporters in $E$. coli shows that SbtA homologs are functional in this heterologous expression system. PLoS One 2014, 9:e115905. 10.1371/journal.pone.0115905

- This study was the first demonstration of negative regulation of a bicarbonate transporter SbtA by its binding partner SbtB. Inferring a role for the latter in bicarbonate uptake regulation.

17. Li HM, Chiu CC: Protein transport into chloroplasts. Annu Rev Plant Biol 2010, 61:157180. 10.1146/annurev-arplant-042809-112222

18. Comai L, Larson-Kelly N, Kiser J, Mau CJ, Pokalsky AR, Shewmaker CK, McBride K, Jones A, Stalker DM: Chloroplast transport of a ribulose bisphosphate carboxylase small subunit-5-enolpyruvyl 3-phosphoshikimate synthase chimeric protein requires part of the mature small subunit in addition to the transit peptide. $J$ Biol Chem 1988, 263:15104-15109.

19. Bionda T, Tillmann B, Simm S, Beilstein K, Ruprecht M, Schleiff E: Chloroplast import signals: the length requirement for translocation in vitro and in vivo. $J \mathrm{Mol} \mathrm{Biol}$ 2010, 402:510-523. 10.1016/j.jmb.2010.07.052

20. Rolland V, Badger MR, Price GD: Redirecting the Cyanobacterial Bicarbonate Transporters BicA and SbtA to the Chloroplast Envelope: Soluble and Membrane Cargos Need Different Chloroplast Targeting Signals in Plants. Frontiers in Plant Science 2016, 7. 10.3389/fpls.2016.00185

21. Uehara S, Adachi F, Ito-Inaba $Y$, Inaba $T$ : Specific and Efficient Targeting of Cyanobacterial Bicarbonate Transporters to the Inner Envelope Membrane of Chloroplasts in Arabidopsis. Front Plant Sci 2016, 7:16. 10.3389/fpls.2016.00016

22. Flugge UI, Hausler RE, Ludewig F, Fischer K: Functional genomics of phosphate antiport systems of plastids. Physiologia Plantarum 2003, 118:475-482.

23. Flügge UI: Transport in and out of plastids: does the outer envelope membrane control the flow? Trends in Plant Science 2000, 5:135-137. 10.1016/s1360-1385(00)01578-8

24. Gutierrez-Carbonell E, Takahashi D, Lattanzio G, Rodriguez-Celma J, Kehr J, Soll J, Philippar $\mathrm{K}$, Uemura M, Abadia J, Lopez-Millan AF: The distinct functional roles of the inner and outer chloroplast envelope of Pea (Pisum sativum) as revealed by proteomic approaches. J Proteome Res 2014, 13:2941-2953. 10.1021/pr500106s

25. Breuers FK, Brautigam A, Geimer S, Welzel UY, Stefano G, Renna L, Brandizzi F, Weber AP: Dynamic Remodeling of the Plastid Envelope Membranes - A Tool for Chloroplast Envelope in vivo Localizations. Front Plant Sci 2012, 3:7. 10.3389/fpls.2012.00007

26. Rosado-Alberio J, Weier TE, Stocking CR: Continuity of the Chloroplast Membrane Systems in Zea mays L. Plant Physiol 1968, 43:1325-1331. 10.1104/pp.43.9.1325

27. Froehlich JE, Keegstra K: The role of the transmembrane domain in determining the targeting of membrane proteins to either the inner envelope or thylakoid membrane. Plant J 2011, 68:844-856. 10.1111/j.1365-313X.2011.04735.x

28. Price GD, Shelden MC, Howitt SM: Membrane topology of the cyanobacterial bicarbonate transporter, SbtA, and identification of potential regulatory loops. Mol Membr Biol 2011, 28:265-275. 10.3109/09687688.2011.593049

29. Shelden MC, Howitt SM, Price GD: Membrane topology of the cyanobacterial bicarbonate transporter, BicA, a member of the SulP (SLC26A) family. Mol Membr Biol 2010, 27:12-23. 10.3109/09687680903400120

30. Cabantous S, Terwilliger TC, Waldo GS: Protein tagging and detection with engineered self-assembling fragments of green fluorescent protein. Nat Biotechnol 2005, 23:102-107. 10.1038/nbt1044 
31. Wiesemann K, Gross LE, Sommer M, Schleiff E, Sommer MS: self-assembling GFP: a versatile tool for plant (membrane) protein analyses. Methods Mol Biol 2013, 1033:131-144. 10.1007/978-1-62703-487-6_9

32. van Dooren GG, Tomova C, Agrawal S, Humbel BM, Striepen B: Toxoplasma gondii Tic20 is essential for apicoplast protein import. Proc Natl Acad Sci U S A 2008, 105:1357413579. 10.1073/pnas.0803862105

33. Sommer MS, Daum B, Gross LE, Weis BL, Mirus O, Abram L, Maier UG, Kuhlbrandt W, Schleiff E: Chloroplast Omp85 proteins change orientation during evolution. Proc Natl Acad Sci U S A 2011, 108:13841-13846. 10.1073/pnas.1108626108

34. Conroy MJ, Durand A, Lupo D, Li XD, Bullough PA, Winkler FK, Merrick M: The crystal structure of the Escherichia coli AmtB-GInK complex reveals how GInK regulates the ammonia channel. Proc Nat Acad Sci USA 2007, 104:1213-1218. $10.1073 /$ pnas.0610348104

35. Atkinson N, Feike D, Mackinder LC, Meyer MT, Griffiths H, Jonikas MC, Smith AM, McCormick AJ: Introducing an algal carbon-concentrating mechanism into higher plants: location and incorporation of key components. Plant Biotechnol J 2015. 10.1111/pbi.12497

-In this study components of an algal CCM were expressed in plants via the nucleus. Although most of them localised in the same compartment both in plants and algae, none of them were functional in plants. This highlights the need to fully understand the activation of CCM components in their plant hosts.

36. Badger MR, Hanson D, Price GD: Evolution and diversity of $\mathrm{CO}_{2}$ concentrating mechanisms in cyanobacteria. Funct Plant Biol 2002, 29:161-173. 10.1071/Pp01213

37. Kinney JN, Axen SD, Kerfeld CA: Comparative analysis of carboxysome shell proteins. Photosynth Res 2011, 109:21-32. 10.1007/s11120-011-9624-6

38. Dou Z, Heinhorst S, Williams E, Murin C, Shively J, Cannon G: $\mathbf{C O}_{2}$ fixation kinetics of Halothiobacillus neapolitanus mutant carboxysomes lacking carbonic anhydrase suggest the shell acts as a diffusional barrier for $\mathbf{C O}_{2}$.J Biol Chem 2008:10377-10384. 10.1074/jbc.M709285200

39. Hauser T, Popilka L, Hartl FU, Hayer-Hartl M: Role of auxiliary proteins in Rubisco biogenesis and function. Nature Plants 2015, 1:1-11. 10.1038/Nplants.2015.65

40. Tsai YC, Lapina MC, Bhushan S, Mueller-Cajar O: Identification and characterization of multiple rubisco activases in chemoautotrophic bacteria. Nat Commun 2015, 6:8883. 10.1038/ncomms9883

-This study identifies CbbQ and CbbO as components of a hetero-oligomeric RuBisCO activase complex from the chemolithoautotroph Acidithiobacillus ferrooxidans, utilising ATP in the remodelling of inhibited RuBisCO.

41. Sutter M, Roberts EW, Gonzalez RC, Bates C, Dawoud S, Landry K, Cannon GC, Heinhorst S, Kerfeld CA: Structural Characterization of a Newly Identified Component of alphaCarboxysomes: The AAA+ Domain Protein CsoCbbQ. Sci Rep 2015, 5:16243. $10.1038 /$ srep 16243

-A recent advance which shows that RuBisCO activase proteins CbbQ and CbbO are part of the carboxysome shell in the canonical $\alpha$-carboxysomal species Halothiobacillus neapolitanus. This suggests a specific role for RuBisCO activases either at the carboxysome shell surface or within; a previously undescribed property of the compartment.

42. Houtz RL, Magnani R, Nayak NR, Dirk LM: Co- and post-translational modifications in Rubisco: unanswered questions. J Exp Bot 2008, 59:1635-1645. 10.1093/jxb/erm360

43. Whitney SM, Birch R, Kelso C, Beck JL, Kapralov MV: Improving recombinant Rubisco biogenesis, plant photosynthesis and growth by coexpressing its ancillary RAF1 chaperone. Proc Natl Acad Sci U S A 2015, 112:3564-3569. 10.1073/pnas.1420536112 
-An important addition to the growing literature on the chaperones needed for correct folding of RuBisCO when expressed in a foreign host, indicating the requirement for cognate RuBisCO accumulation factor (RAF1) to enhance soluble RuBisCO.

44. Wheatley NM, Sundberg CD, Gidaniyan SD, Cascio D, Yeates TO: Structure and identification of a pterin dehydratase-like protein as a RuBisCO assembly factor in the alpha-carboxysome. J Biol Chem 2014, 289:7973-7981. 10.1074/jbc.M113.531236

45. Mueller-Cajar O, Stotz M, Wendler P, Hartl FU, Bracher A, Hayer-Hartl M: Structure and function of the AAA(+) protein CbbX, a red-type Rubisco activase. Nature 2011, 479:194-U166. 10.1038/nature10568

46. Li LA, Zianni MR, Tabita FR: Inactivation of the monocistronic rca gene in Anabaena variabilis suggests a physiological ribulose bisphosphate carboxylase/oxygenase activase-like function in heterocystous cyanobacteria. Plant Mol Biol 1999, 40:467478. 10.1023/A:1006251808625

47. Peña KL, Castel SE, de Araujo C, Espie GS, Kimber MS: Structural basis of the oxidative activation of the carboxysomal gamma-carbonic anhydrase, CcmM. Proc Nat Acad Sci USA 2010, 107:2455-2460. 10.1073/pnas.0910866107

48. Cai F, Dou Z, Bernstein SL, Leverenz R, Williams EB, Heinhorst S, Shively J, Cannon GC, Kerfeld CA: Advances in Understanding Carboxysome Assembly in Prochlorococcus and Synechococcus Implicate CsoS2 as a Critical Component. Life (Basel) 2015, 5:1141-1171. 10.3390/life5021141

49. Chaijarasphong T, Nichols RJ, Kortright. KE, Nixon CF, Teng PK, Oltrogge LM, Savage DF: Programmed ribosomal frameshifting mediates expression of the $\alpha$-carboxysome. J Mol Biol 2015. 10.1016/j.jmb.2015.11.017

$\bullet \bullet$ A long hoped for description of the mechanism which results in the construction of the two forms of the CsoS2 protein from a single gene. The paper describes a translational frame shift which results in an early stop codon outside the normal coding sequence, thus producing either a full-length protein or one with an apparent C-terminal truncation.

50. Long BM, Badger MR, Whitney SM, Price GD: Analysis of carboxysomes from Synechococcus PCC7942 reveals multiple Rubisco complexes with carboxysomal proteins CcmM and CcaA. J Biol Chem 2007, 282:29323-29335. 10.1074/jbc.M703896200

51. Cot SSW, So AKC, Espie GS: A multiprotein bicarbonate dehydration complex essential to carboxysome function in cyanobacteria. J Bacteriol 2008, 190:936-945. 10.1128/JB.01283-07

52. Kinney JN, Salmeen A, Cai F, Kerfeld CA: Elucidating essential role of conserved carboxysomal protein $\mathrm{CcmN}$ reveals common feature of bacterial microcompartment assembly. $J$ Biol Chem 2012, 287:17729-17736. 10.1074/jbc.M112.355305

53. Long BM, Rae BD, Badger MR, Price GD: Over-expression of the ß-carboxysomal CcmM protein in Synechococcus PCC7942 reveals a tight co-regulation of carboxysomal carbonic anhydrase (CcaA) and M58 content. Photosynth Res 2011, 109:33-45. 10.1007/s11120-011-9659-8

54. Long BM, Tucker L, Badger MR, Price GD: Functional Cyanobacterial $\beta$-Carboxysomes Have an Absolute Requirement for Both Long and Short Forms of the CcmM Protein. Plant Physiol 2010, 153:285-293. 10.1104/pp.110.154948

55. Chen AH, Robinson-Mosher A, Savage DF, Silver PA, Polka JK: The bacterial carbon-fixing organelle is formed by shell envelopment of preassembled cargo. PLOS ONE 2013, 8:e76127. 10.1371/journal.pone.0076127

- This work outlined the assembly pathway of $\beta$-carboxysomes. From the initial structures, up to the final shell closure and redox trigger for CA activity. 
56. Whitney SM, Sharwood RE: Construction of a tobacco master line to improve Rubisco engineering in chloroplasts. J Exp Bot 2008, 59:1909-1921. 10.1093/jxb/erm311

57. Gonzalez-Esquer CR, Shubitowski TB, Kerfeld CA: Streamlined Construction of the Cyanobacterial $\mathrm{CO}_{2}$-Fixing Organelle via Protein Domain Fusions for Use in Plant Synthetic Biology. Plant Cell 2015, 27:2637-2644. 10.1105/tpc.15.00329

- This important advance investigated the construction of carboxysomes via the synthetic merging of important interacting protein domains into one gene, thus minimizing the genetic burden of carboxysome synthesis. While functionality was diminished, this is an important step toward the construction of completely synthetic bacterial micro-compartments.

58. Roberts EW, Cai F, Kerfeld CA, Cannon GC, Heinhorst S: Isolation and characterization of the Prochlorococcus carboxysome reveal the presence of the novel shell protein CsoS1D. J Bacteriol 2012, 194:787-795. 10.1128/JB.06444-11

59. Whitney SM, Sharwood RE: Linked Rubisco subunits can assemble into functional oligomers without impeding catalytic performance. J Biol Chem 2007, 282:38093818. 10.1074/jbc.M610479200

60. Badger MR, Bek EJ: Multiple Rubisco forms in proteobacteria: their functional significance in relation to $\mathrm{CO}_{2}$ acquisition by the CBB cycle. J Exp Bot 2008, 59:15251541. 10.1093/jxb/erm297

61. Scott KM, Henn-Sax M, Harmer TL, Longo DL, Frame CH, Cavanaugh CM: Kinetic isotope effect and biochemical characterization of form IA RubisCo from the marine cyanobacterium Prochlorococcus marinus MIT9313. Limnol and Oceanogr 2007, 52:2199-2204. 10.4319/lo.2007.52.5.2199

62. Whitehead L, Long B, Price D, Badger MR: Comparing the in vivo function of $\alpha$ and $\boldsymbol{\beta}$ carboxysomes in two model cyanobacteria. Plant Physiol 2014, 165:398-411. $10.1104 / p p .114 .237941$

-A comparison of carboxysomes from two separate cyanobacterial lineages which shows that despite their evolutionary disparity, carboxysome performance of both $\alpha$ and $\beta$-carboxysomes is remarkably similar.

63. Badger MR: Kinetic-properties of ribulose 1,5-bisphosphate carboxylase-oxygenase from Anabaena variabilis. Arch Biochem Biophys 1980, 201:247-254. 10.1016/00039861(80)90509-3

64. Hopkinson BM, Young JN, Tansik AL, Binder BJ: The Minimal $\mathbf{C O}_{2}$-Concentrating Mechanism of Prochlorococcus spp. MED4 Is Effective and Efficient. Plant Physiol 2014, 166:2205-2217. 10.1104/pp.114.247049

65. Greene DN, Whitney SM, Matsumura I: Artificially evolved Synechococcus PCC6301 Rubisco variants exhibit improvements in folding and catalytic efficiency. Biochem J 2007, 404:517-524. 10.1042/BJ20070071

66. Rae BD, Forster $\mathrm{B}$, Badger $\mathrm{MR}$, Price GD: The $\mathbf{C O}_{2}$-concentrating mechanism of Synechococcus WH5701 is composed of native and horizontally-acquired components. Photosynth Res 2011, 109:59-72. 10.1007/s11120-011-9641-5

67. Long BM, Price GD, Badger MR: Proteomic assessment of an established technique for carboxysome enrichment from Synechococcus PCC7942. Can J Bot 2005, 83:746-757. 10.1139/B05-058

68. Bonacci W, Teng PK, Afonso B, Niederholtmeyer H, Grob P, Silver PA, Savage DF: Modularity of a carbon-fixing protein organelle. Proc Natl Acad Sci USA 2012, 109:478-483. $10.1073 /$ pnas.1108557109

69. Price GD, von Caemmerer S, Evans JR, Yu JW, Lloyd J, Oja V, Kell P, Harrison K, Gallagher A, Badger MR: Specific Reduction of Chloroplast Carbonic-Anhydrase Activity by Antisense Rna in Transgenic Tobacco Plants Has a Minor Effect on Photosynthetic Co2 Assimilation. Planta 1994, 193:331-340. 10.1007/BF00201810 
469 Figure 1. The CCM of cyanobacteria. Protein components discussed in this review are 470 highlighted, whilst those outside the expected chloroplast engineering strategy are shown in 471 grey. The cyanobacterial CCM utilises three known bicarbonate transporters: BicA (magenta), 472 SbtA (purple), and the BCT1 complex (an ATP-binding cassette transporter comprising Cmp A, $473 \mathrm{~B}, \mathrm{C}$ and D subunits). Further, two thylakoid-bound $\mathrm{CO}_{2}$ uptake complexes recover cytoplasmic $474 \mathrm{CO}_{2}: \mathrm{NDH}-\mathrm{I}_{3}$, and NDH-I $\mathrm{I}_{4}$. These are supported by at least three ion exchangers which recover the chemical gradients energising active $\mathrm{Ci}$-uptake: $\mathrm{Na}^{+} / \mathrm{H}^{+}$antiporter NhaS3 (blue); and PxcA and Mnh complexes exporting $\mathrm{H}^{+}$. The potential role of the companion protein SbtB (light purple) in SbtA activation is indicated. The accumulated bicarbonate pool is utilised within the carboxysome (yellow), where bicarbonate is dehydrated to $\mathrm{CO}_{2}$ and fixed into organic carbon by RuBisCO in the Calvin-Benson-Bassham cycle (CBB). Low rate, diffusive movement of $\mathrm{CO}_{2}$ into the cell is indicated on the left of the figure. $\mathrm{CO}_{2}$ uptake complexes also play a role in scavenging of $\mathrm{CO}_{2}$ lost from carboxysomes. Locations of the proteins and structures within the cell are indicated in Figure 2.

Figure 2. Engineering a $\mathbf{C}_{3}$ Chloroplastic CCM. Mathematical models suggest that the ideal engineering trajectory occurs in three general stages $[5,6]$. 1. Active bicarbonate transporters [BicA (magenta), SbtA (purple)] from cyanobacteria are introduced into the chloroplast inner membrane within a C3 plant cell (brown); subsequently, ancillary $\mathrm{Na}^{+} / \mathrm{H}^{+}$pumps $\left[\mathrm{Na}^{+} / \mathrm{H}^{+}\right.$ antiporter NhaS3 (blue)]. 2. Carboxysomes (yellow) with their cognate RuBisCO are introduced. 3. Stromal carbonic anhydrase (CA) enzymes, as well as the endogenous RuBisCO, are eliminated and carboxysomes and transporters are combined in the one chloroplast. Genetic removal of endogenous chloroplastic the RuBisCO LSU gene can be carried out during incorporation of carboxysomal RuBisCO via recombination [14]. The genetic excision or downregulation of stromal CA has little effect on photosynthetic performance in WT plants [69] and can therefore be carried out at any time. However, the existence of stromal CA will short-cut a chloroplastic CCM by dissipating the stromal bicarbonate pool as $\mathrm{CO}_{2}$ [5] and must at least be a final step in the process before maximal advantage of the CCM can be seen. Further steps are likely required for fine-tuning of the system. 
501 the RuBisCO large and small subunit genes; purple are known or putative RuBisCO activases

502 and chaperones; magenta are carboxysomal carbonic anhydrases (CA); blue are outer shell 503 proteins including vertex proteins; and yellow are structural proteins (Table 1). A. $\alpha$ 504 Carboxysomes from the gammaproteobacterium Halothiobacillus neapolitanus $\mathrm{C} 2$ are the best 505 understood of the $\alpha$-type, and utilise the acRAF chaperone [r, 44], and CbbQ/O activase [41]. B. 506 A comparatively simple $\alpha$-carboxysome operon from Prochlorococcus marinus, utilising the 507 acRAF (r) chaperone only, although its absolute requirement is unknown. Other cyanobacterial $508 \alpha$-carboxysomes appear to also utilise a CbbX activase [58]. C. Genetic components encoding the 509 best understood $\beta$-carboxysome from Synechococcus elongatus PCC 7942, utilising the putative 510 chaperone $\operatorname{RbcX}(\mathrm{X})$, and the additional carboxysomal CA CcaA. D. A comparatively simple $\beta$ 511 carboxysome operon from Gloeobacter violaceus PCC 7421, utilising the RuBisCO activase Rca, 512 and the CcmM protein both as a structural component and its N-terminal CA [47]. 


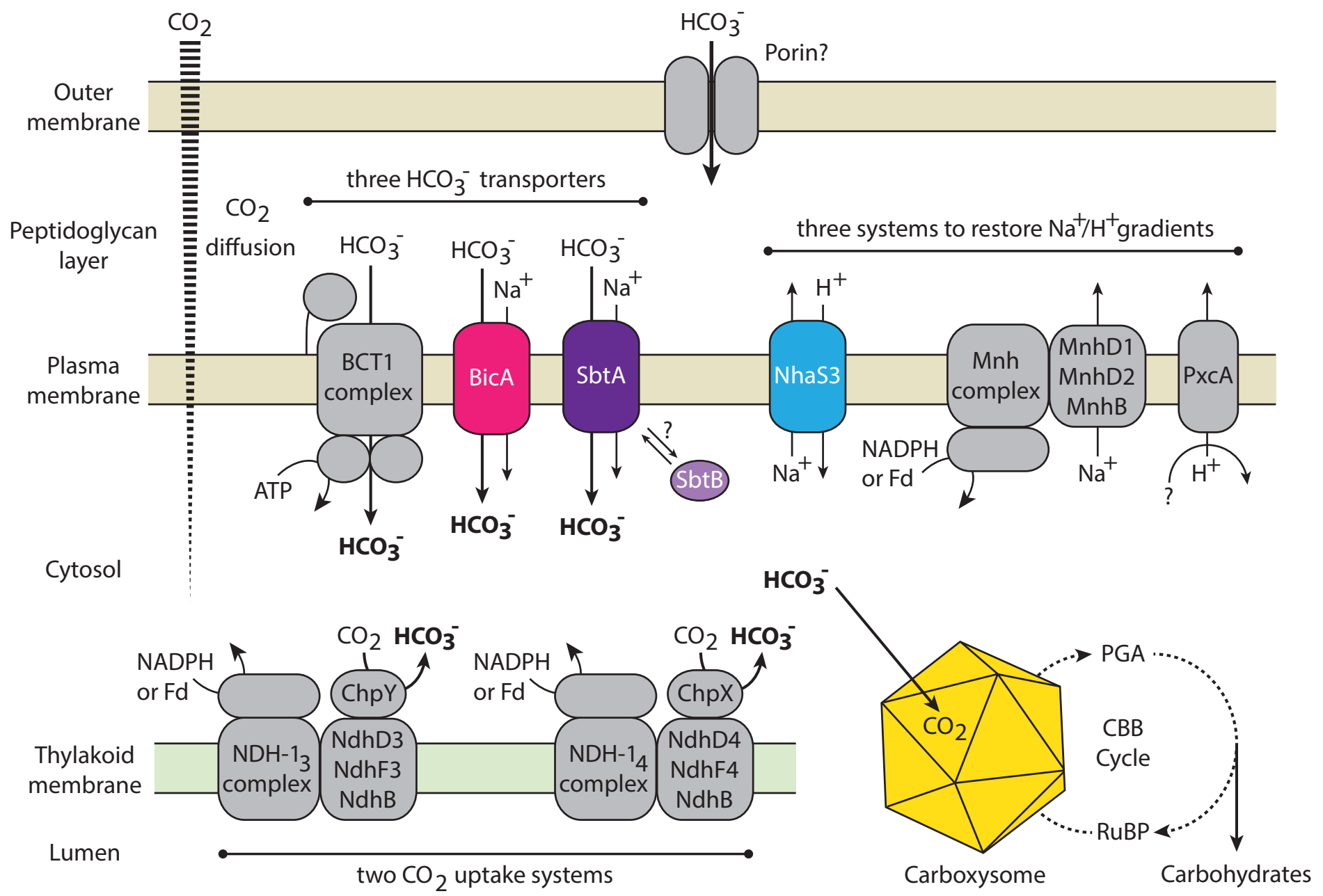

Figure 1 


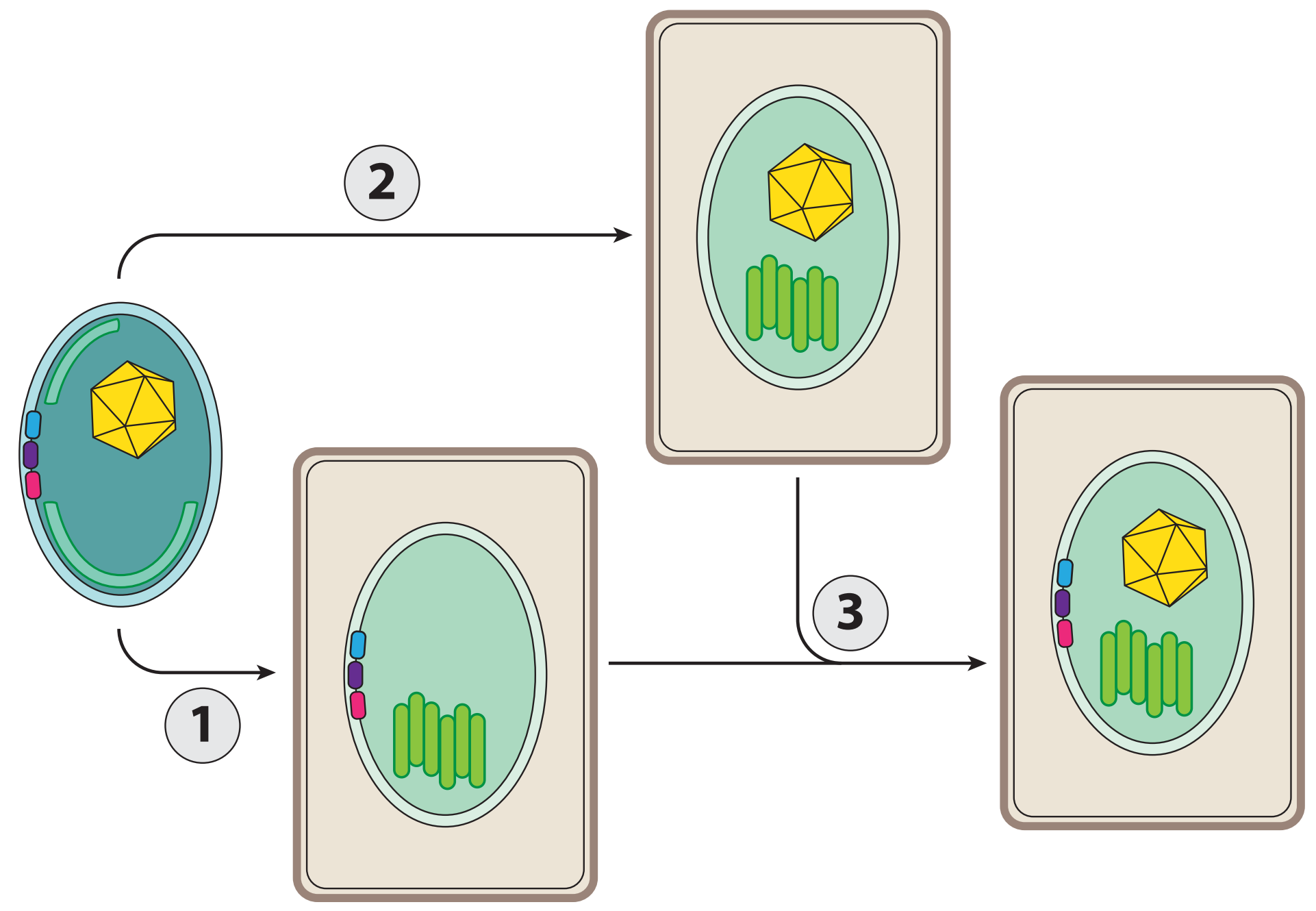

Figure 2 
A- Halothiobacillus neapolitanus C2

csoS4. csoS1

$c b b l$

B- Prochlorococcus marinus subsp. pastoris MED4

S1D -1 (S)

C- Synechococcus elongatus PCC 7942

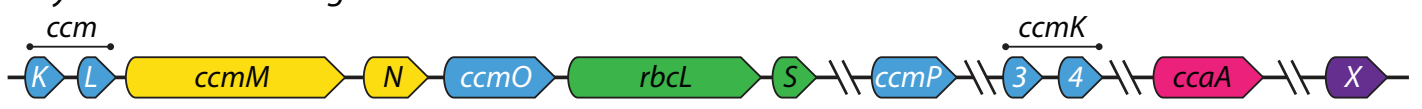

D- Gloeobacter violaceus PCC 7421

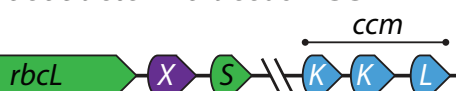

$\mathrm{ccmM}>\mathrm{N}-\mathrm{ccmO}-\mathrm{H}-\mathrm{rca}-\mathrm{N}-\mathrm{ccmP}-$

Figure 3 
(2)

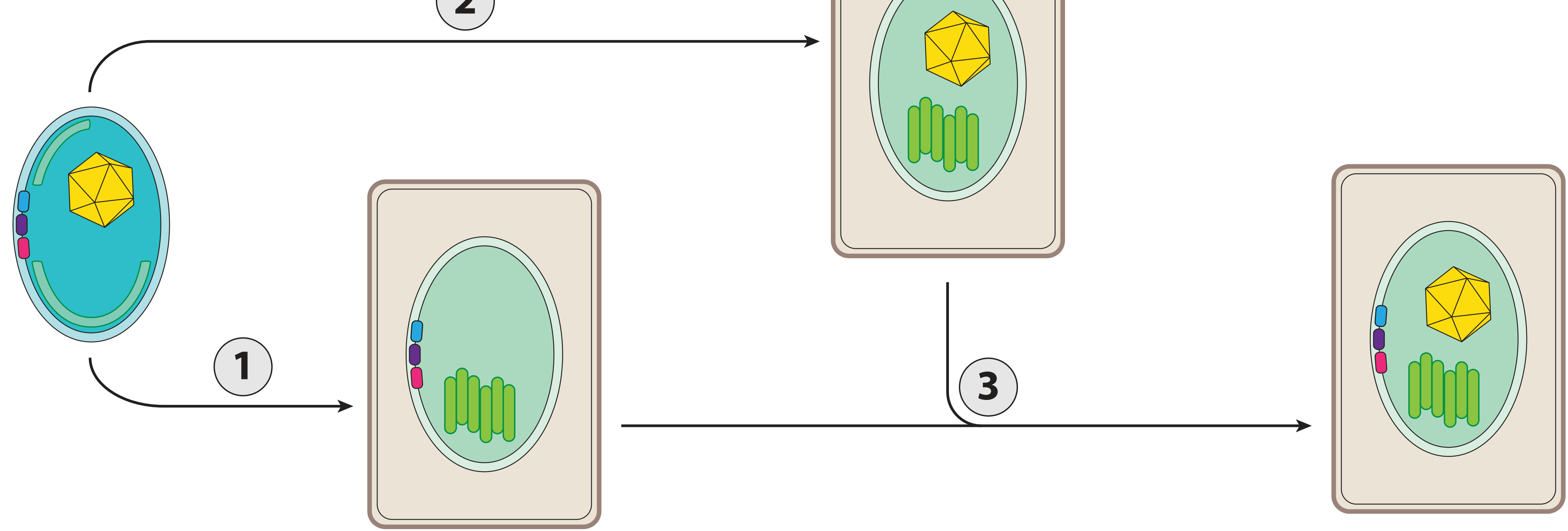

